\title{
Study of Release-Retardant Polymers in Formulation Development of Cinnarizine-HCl Sustained-release Oral Suspension Using Raft Technology
}

\author{
Tabinda Islam, Nusrat Hossain, Mohsina Rahman, Sadia Shabnam, Eyasmin \\ Chowdhury, Syeda Tahsin Nahar, Zarin Tasnim Gias and Hasan Mahmud Reza
}

\author{
Department of Pharmaceutical Sciences, School of Health and Life Sciences \\ North South University, Plot-15, Block-B, Bashundhara, Dhaka-1229, Bangladesh
}

(Received: September 11, 2019; Accepted: February 24, 2020; Published (web): March 4, 2020)

\begin{abstract}
The main objective of this research was to develop a sustained-release suspension of cinnarizine hydrochloride using raft-forming technique. This innovative approach has been utilized to formulate a series of suspension formulations using hydroxypropyl cellulose (HPC) as a release-retardant polymeric agent. Cinnarizine sustained-release suspensions were prepared by physical mixing method with varying concentrations and combinations of HPC, sodium citrate, sodium saccharin, calcium carbonate, sodium alginate, methyl hydroxybenzoate and propyl hydroxybenzoate. The formulations were subjected for determination of floating time, floating lag time, weight of the raft, physical appearance and in-vitro dissolution. The dissolution was conducted through USP apparatus 2 (paddle type) in $0.1 \mathrm{~N}$ hydrochloric acid medium having $\mathrm{pH} 1.2$. The key findings of the study demonstrate that a stable sustained-release suspension of cinnarizine can be formulated using raft-forming approach for increased bioavailability and patient-convenience.
\end{abstract}

Key words: Cinnarizine-HCl, raft-forming method, sustained-release drug delivery, in-vitro evaluation, dissolution study.

\section{INTRODUCTION}

Oral controlled-release dosage forms have gained much attention of the researchers worldwide due to its ability to maintain an effective drug concentration in the blood for a longer period and thus it offers numerous therapeutic advantages e.g. reducing the dosing interval, ease of administration as well as high patient compliance. ${ }^{1,2}$ On the other hand, considering the gastrointestinal (GI) retention and emptying time, oral route of pharmaceutical dosage form could be a problem since physiological conditions are different among individuals. Therefore, it was an urge for the researchers to design such dosage forms that could remain in the stomach for a prolonged time to significantly increase the gastric residence/retention time for enhanced bioavailability and better therapeutic outcome. ${ }^{2}$

Correspondence to: Hasan Mahmud Reza

E-mail: hasan.reza@northsouth.edu; Phone: +880-1715885651

Dhaka Univ. J. Pharm. Sci. 19(1): 15-24, 2020 (June) DOI: https://doi.org/10.3329/dujps.v19i1.47814
Sustained-release drug delivery system is based on the important consideration in designing a dosage form is to achieve a predictable therapeutic response and minimize the possible toxic effects of the drug. This target is usually achieved by attempting to design a formulation that follows a zero-order release pattern of the drug from the formulation bulk or dosage form. ${ }^{3,4}$

The type and concentration of polymers used in sustained-release formulation plays a crucial role in establishing a desired drug-release pattern. Generally, the incorporation of one or more release-retarding polymers with the drug molecule serves as the basis for formulating time-dependent/extended/sustainedrelease dosage forms. ${ }^{5}$ Nevertheless, conventional sustained-release drug delivery systems are usually formulated in such way that the drug release follows a "slow-first-order" release pattern which mimics the zero-order kinetics but they are concentrationdependent. Gastro-retentive system has been 
developed by employing the technology that facilitates an enhanced gastric retention time for the sustained-release dosage form to increase drug bioavailability. ${ }^{5,6}$

A more recent development in the area of pharmaceutical dosage forms and drug delivery system is the use of raft technology. The conventional use of "Rafts" is as a support for swimmers due to its structural feasibility to float over the water for a longer period. ${ }^{6,7}$ Hence, pharmaceutical scientists have designed such a carrier system that can float over the GI fluid/content. The key mechanism in rafts formation in pharmaceutical preparations involve the formation of a viscous gel-like content when the gel-forming agents come in contact with GI fluid and become swelled to make a few layers of rafts. ${ }^{7}$ The preparations usually contain alkaline substances that form $\mathrm{CO}_{2}$ gas and make the rafts float due to having very low bulk density. Nevertheless, among various gel-forming agents, alginates are most popular due to its high thickening, gel-forming, and stabilizing properties. Alginate-based raft-forming formulations mostly contain $\mathrm{CO}_{2}$ forming bicarbonates that facilitate the floating capacity of the rafts and provide longer action. There are several advantages of raftforming technology in pharmaceutical formulations such as- rapid and long-term action of the drug, better patient compliance and no interaction with the digestive process. ${ }^{7,8}$

Cinnarizine (1-(Diphenylmethyl)-4-(3-phenyl-2propenyl) piperazine) is usually prescribed in the management of vestibular and associated symptoms. However, cinnarizine hydrochloride belongs to the labyrinthine sedative and peripheral antivasoconstrictor class of drugs that acts directly in both peripheral and central origin of the central nervous system. ${ }^{9}$ Cinnarizine hydrchloride shows antihistaminic activity by selectively blocking the $\mathrm{H} 1$ receptors and inhibiting the influx of calcium ions intracellularly. ${ }^{9,10}$ However, due to poor solubility profile of cinnarizine hydrchloride, this molecule is classified under biopharmaceutical classification system (BCS) class-II system which indicates low aqueous solubility and high membrane permeability; thus, dissolution is the rate-limiting step. ${ }^{10}$ Therefore, this class of drug needs advanced research methodologies and molecular optimization to overcome the existing obstacles and to develop effective therapeutic options over the conventional formulations. ${ }^{10}$

The basic aim of this study was to develop raftforming formulations of cinnarizine hydrchloride in a form of oral suspension using release-retardant polymers in combination with alginate-based gelforming agents. The study was conducted to evaluate the effectiveness of formulation variables on the following responses: raft stability and strength, floating time and capacity, and in-vitro drug release at 12-hours sustained time period.

\section{MATERIALS AND METHODS}

\section{Materials}

Cinnarizine hydrochloride was obtained as a gift sample from ACI pharmaceuticals Ltd. (Dhaka, Bangladesh). Sodium alginate, hydroxypropyl cellulose, $\mathrm{HCl}$, calcium carbonate, sodium citrate, sodium saccharin, ethanol, and methanol were purchased commercially.

\section{Methods}

Preparation of cinnarizine hydrochloride suspensions. Drug, release-retardant polymers and other additives were weighed accurately. All ingredients apart from the release-retardant polymers and drug were dissolved in sufficient quantities of Milli-Q water to make a uniform solution. Methyl hydroxybenzoate, propyl hydroxybenzoate, and sodium alginate were added to the solution followed by adequate heating and rigorous stirring to achieve a viscous dispersion. The dispersion was allowed to cool to $\sim 30^{\circ} \mathrm{C}$ and the subsequent preparation of the suspension was carried out by adding the cinnarizine hydrochloride dispersion (prepared separately) and HPC. To avoid any lumps, an accurately weighed calcium carbonate was added to the suspension and the final volume was made using Milli-Q water; sufficient stirring was done to prepare a homogenous 
suspension. A total 30 formulations of cinnarizine hydrochloride suspension were prepared contained a varying concentration of the gel-forming agents (e.g. sodium alginate, hydroxypropyl cellulose and calcium carbonate (Table 1). ${ }^{11,12}$

Table 1. Formulation design of cinnarizine hydrochloride sustained-release suspension

\begin{tabular}{|c|c|c|c|}
\hline $\begin{array}{l}\text { Formulation } \\
\text { number }(\mathrm{R})\end{array}$ & $\begin{array}{l}\text { Sodium alginate } \\
(\%)\end{array}$ & $\begin{array}{l}\text { Hydroxypropyl cellulose } \\
(\%)\end{array}$ & $\begin{array}{c}\text { Calcium carbonate } \\
(\%)\end{array}$ \\
\hline R-1 & 8.000 & 5.000 & 6.000 \\
\hline R-2 & 8.000 & 4.500 & 6.000 \\
\hline R-3 & 8.000 & 4.850 & 6.000 \\
\hline $\mathrm{R}-4$ & 8.000 & 3.500 & 6.000 \\
\hline $\mathrm{R}-5$ & 8.000 & 3.000 & 7.000 \\
\hline R-6 & 8.000 & 3.000 & 8.000 \\
\hline R-7 & 8.000 & 3.000 & 5.000 \\
\hline R-8 & 8.000 & 2.500 & 6.000 \\
\hline R-9 & 7.000 & 3.000 & 6.000 \\
\hline R-10 & 9.000 & 3.000 & 6.000 \\
\hline $\mathrm{R}-11$ & 8.000 & 1.000 & 6.000 \\
\hline $\mathrm{R}-12$ & 8.000 & 1.250 & 6.000 \\
\hline $\mathrm{R}-13$ & 8.000 & 4.250 & 6.000 \\
\hline R-14 & 8.000 & 1.750 & 6.000 \\
\hline $\mathrm{R}-15$ & 8.000 & 2.000 & 6.000 \\
\hline R-16 & 8.000 & 2.250 & 6.000 \\
\hline $\mathrm{R}-17$ & 8.000 & 2.750 & 6.000 \\
\hline $\mathrm{R}-18$ & 8.000 & 4.500 & 6.000 \\
\hline R-19 & 8.000 & 3.250 & 6.000 \\
\hline $\mathrm{R}-20$ & 8.000 & 3.750 & 6.000 \\
\hline $\mathrm{R}-21$ & 8.000 & 4.250 & 6.000 \\
\hline $\mathrm{R}-22$ & 8.000 & 4.750 & 6.000 \\
\hline $\mathrm{R}-23$ & 8.000 & 4.000 & 7.000 \\
\hline $\mathrm{R}-24$ & 8.000 & 1.500 & 7.000 \\
\hline $\mathrm{R}-25$ & 8.000 & 2.000 & 7.000 \\
\hline R-26 & 8.000 & 2.500 & 7.000 \\
\hline $\mathrm{R}-27$ & 8.000 & 2.750 & 7.000 \\
\hline $\mathrm{R}-28$ & 8.000 & 3.500 & 7.000 \\
\hline $\mathrm{R}-29$ & 8.000 & 4.000 & 7.000 \\
\hline R-30 & 8.000 & 4.500 & 7.000 \\
\hline
\end{tabular}

Physical appearance. The appearance and colour of the suspension formulations were inspected visually by taking each formulation in separate prelabelled falcon tubes.

Sedimentation volume. The sedimentation volume of the suspension formulations was inspected in preset time intervals during a 15-day storage period to a constant standing. The data was recorded in terms of the ratio of the ultimately settled height
$\mathrm{(Hu}$ ) to the original height $(\mathrm{Ho})$ of the suspension in a $100 \mathrm{ml}$ measuring cylinder and was expressed using the following equation, $\mathrm{F}$ (sedimentation rate) = $\mathrm{Hu} / \mathrm{H}_{0}{ }^{13,14}$

Degree of flocculation. The volume of flocculated suspension was measured at a 15-day time without any shaking/agitation. The value was measured using the following formula, $B=F / F \infty=$ $(\mathrm{Vu} / \mathrm{Vo}) /(\mathrm{V} \infty / \mathrm{Vo}) \times 100$ where, $B$ is the ratio of the 
sedimentation volume of the flocculated suspension, $\mathrm{F}$ to the sedimentation volume of the deflocculated suspension, $\mathrm{F}_{\infty} .{ }^{14}$

\section{Resuspendability/redispersibility.}

Redispersibility was measured through manual shaking of each experimental suspension bottles after a 4-day constant standing time at room temperature. The time required for the sediment to convert into a homogenous suspension was recorded. ${ }^{15}$

Floating lag time. Floating lag time was determined by measuring the time taken for the suspensions to emerge onto the surface of dissolution medium $\left(0.1 \mathrm{~N} \mathrm{HCl}, \mathrm{pH} 1.2\right.$, at $\left.37^{\circ} \mathrm{C}\right)$ and was expressed in seconds. ${ }^{15,16}$

Floating time/buoyancy. This test is performed by recording the time required for the each suspension formulation to remain floated over the dissolution media $\left(0.1 \mathrm{~N} \mathrm{HCl}, \mathrm{pH} 1.2\right.$, at $\left.37^{\circ} \mathrm{C}\right)$. The values were expressed in hours. ${ }^{15,16}$

Raft weight/strength. An amount of each suspension equivalent to the unit dose was transferred to a $100 \mathrm{ml}$ beaker prefilled with $0.1 \mathrm{~N} \mathrm{HCl}$ at a constant temperature of $37^{\circ} \mathrm{C}$. An $\mathrm{L}$ shaped wire probe was held upright in the beaker throughout the whole period (approx. $30 \mathrm{~min}$ ) for the raft development. Once the suspension was poured into the beaker, the raft started forming around the wire probe. Water was added drop-wise to the left pan and the weight of water required to break the raft was recorded. ${ }^{15,16-18}$

In vitro drug release. The test was carried out using the USP apparatus II prefilled with $900 \mathrm{ml}$ of dissolution medium $(0.1 \mathrm{~N} \mathrm{HCl})$, which was allowed to equilibrate at a temperature of $37 \pm 5^{\circ} \mathrm{C}$, having an ionic strength similar to the gastrointestinal tract (e.g. $\mathrm{pH}$ 1.2). The paddle rotation was set at $50 \mathrm{rpm}$ for the whole dissolution period. A $15 \mathrm{ml}$ suspension was carefully measured placed at the bottom of each dissolution vessel with the help of a glass pipette. A 5 $\mathrm{ml}$ aliquot was withdrawn from each dissolution medium at preset intervals of 15 minutes, 30 minutes, $1,2,4,6,8$ and 12 hours followed by a replacement with an equivalent amount of fresh dissolution medium to maintain the sink condition. The amount
(\%) of cinnarizine hydrochloride release was measured using UV- spectrophotometer at $\lambda_{\max } 251$ $\mathrm{nm}$ after filtration and necessary dilution of the dissolution samples. ${ }^{15-18}$

Statistical analysis. The dissolution data were analysed by one-way analysis of variance (ANOVA) followed by Tukey's multiple comparisons test using GraphPad Prism version 8.00. A comparative dissolution study was done for the following formulations R1, R3, R13, R17, R23 and R27 and the differences of cumulative percentage of drug release were considered significant when $p<0.05$. $^{25}$

\section{RESULTS AND DISCUSSION}

The formulations were evaluated for physical appearance, floating lag time/ in-vitro gelation time, floating time, redispersibility, raft weight, sedimentation volume and in vitro-drug release characteristics (Table 2). All formulations were readily redispersible. Cake-like sediment was found on standing in few formulations, however, upon reagitation, the sediment was found to be redispersible which complied with the monographic requirement of resuspendability for suspension formulations. Based on the results obtained shown in (Table 2), further tests were performed.

Floating lag time. Floating lag time of the formulations is shown in (Table 2). The formulation exhibiting the lowest floating lag time of 25 seconds was found with R9 which contained $2.75 \%$ hydroxypropyl cellulose. The formulation with the highest floating lag time of 3 minutes 55 seconds was found with R8 which contained $2.00 \%$ hydroxypropyl cellulose. The formulation with the highest percentage of hydroxypropyl cellulose, R1 (5\%), produced the second highest floating lag time of 3 minutes 50 seconds. However, the formulations with the lowest percentage of hydroxypropyl cellulose, R11 and R23 (1.0\%), produced moderate floating lag times of 1 minute 47 seconds and 1 minute 42 seconds, respectively. From the above observations, it is clear that the floating lag time is not affected by the percentage of release-retardant polymer. ${ }^{15,16}$ 
Floating time/buoyancy. Almost all the formulations produced similar floating time or raft residence time (15 16 hours) (Table 2) which indicated that the presence of release-retardant polymer hinders the erosion of the raft and causes a prolonged floating time. ${ }^{15,16}$

Table 2. Physical appearance, floating lag time, floating time and raft weight of cinnarizine hydrochloride sustained-release suspensions.

\begin{tabular}{|c|c|c|c|c|}
\hline Formulation no. & $\begin{array}{c}\text { Physical } \\
\text { appearance }\end{array}$ & Floating lag time & $\begin{array}{l}\text { Floating time } \\
\quad \text { (hour) }\end{array}$ & Raft wt. (g) \\
\hline $\mathrm{R}-1$ & pass & $3 \mathrm{~min} 50 \mathrm{sec}$. & $18.1 \pm 0.06$ & $3.24 \pm 0.02$ \\
\hline $\mathrm{R}-2$ & pass & $2 \mathrm{~min} 52 \mathrm{sec}$. & $15.2 \pm 0.20$ & $3.16 \pm 0.03$ \\
\hline $\mathrm{R}-3$ & pass & $3 \min 49 \mathrm{sec}$. & $16.1 \pm 0.12$ & $3.20 \pm 0.02$ \\
\hline $\mathrm{R}-4$ & pass & $3 \mathrm{~min} 32 \mathrm{sec}$. & $15.1 \pm 0.03$ & $3.01 \pm 0.01$ \\
\hline $\mathrm{R}-5$ & pass & $2 \min 37 \mathrm{sec}$. & $16.2 \pm 0.06$ & $3.02 \pm 0.01$ \\
\hline R-6 & pass & $3 \mathrm{~min} 45 \mathrm{sec}$. & $15.1 \pm 0.02$ & $3.05 \pm 0.04$ \\
\hline $\mathrm{R}-7$ & pass & $3 \mathrm{~min} 67 \mathrm{sec}$. & $16.2 \pm 0.07$ & $2.96 \pm 0.02$ \\
\hline $\mathrm{R}-8$ & pass & $3 \min 23 \mathrm{sec}$. & $16.1 \pm 0.03$ & $3.10 \pm 0.02$ \\
\hline R-9 & pass & $25 \mathrm{sec}$ & $8.1 \pm 0.09$ & $3.07 \pm 0.02$ \\
\hline $\mathrm{R}-10$ & pass & $2 \mathrm{~min} 45 \mathrm{sec}$. & $15.2 \pm 0.04$ & $3.01 \pm 0.01$ \\
\hline R-11 & pass & $1 \mathrm{~min} 47 \mathrm{sec}$. & $16.1 \pm 0.03$ & $1.97 \pm 0.01$ \\
\hline $\mathrm{R}-12$ & pass & $3 \mathrm{~min} 50 \mathrm{sec}$. & $16.1 \pm 0.05$ & $2.14 \pm 0.04$ \\
\hline $\mathrm{R}-13$ & pass & $3 \mathrm{~min} 50 \mathrm{sec}$. & $15.1 \pm 0.03$ & $3.18 \pm 0.03$ \\
\hline $\mathrm{R}-14$ & pass & $3 \mathrm{~min} 50 \mathrm{sec}$. & $16.2 \pm 0.03$ & $2.37 \pm 0.02$ \\
\hline $\mathrm{R}-15$ & pass & $3 \mathrm{~min} 50 \mathrm{sec}$. & $15.2 \pm 0.03$ & $2.38 \pm 0.01$ \\
\hline $\mathrm{R}-16$ & pass & $3 \mathrm{~min} 12 \mathrm{sec}$. & $16.2 \pm 0.06$ & $2.42 \pm 0.04$ \\
\hline $\mathrm{R}-17$ & pass & $3 \mathrm{~min} 53 \mathrm{sec}$. & $15.2 \pm 0.03$ & $3.17 \pm 0.05$ \\
\hline $\mathrm{R}-18$ & pass & $3 \mathrm{~min} 19 \mathrm{sec}$. & $16.1 \pm 0.02$ & $2.51 \pm 0.02$ \\
\hline $\mathrm{R}-19$ & pass & $2 \mathrm{~min} 30 \mathrm{sec}$. & $15.2 \pm 0.03$ & $3.07 \pm 0.02$ \\
\hline $\mathrm{R}-20$ & pass & $1 \mathrm{~min} 42 \mathrm{sec}$. & $16.4 \pm 0.03$ & $3.10 \pm 0.01$ \\
\hline $\mathrm{R}-21$ & pass & $3 \mathrm{~min} 12 \mathrm{sec}$. & $16.1 \pm 0.07$ & $3.17 \pm 0.01$ \\
\hline $\mathrm{R}-22$ & pass & $3 \mathrm{~min} 37 \mathrm{sec}$. & $16.1 \pm 0.02$ & $3.20 \pm 0.01$ \\
\hline $\mathrm{R}-23$ & pass & $3 \mathrm{~min} 42 \mathrm{sec}$. & $15.1 \pm 0.02$ & $3.18 \pm 0.04$ \\
\hline $\mathrm{R}-24$ & pass & $3 \mathrm{~min} 16 \mathrm{sec}$. & $16.1 \pm 0.03$ & $2.21 \pm 0.02$ \\
\hline $\mathrm{R}-25$ & pass & $2 \min 34 \mathrm{sec}$ & $15.2 \pm 0.02$ & $2.34 \pm 0.03$ \\
\hline R-26 & pass & $3 \mathrm{~min} 11 \mathrm{sec}$. & $16.2 \pm 0.03$ & $2.50 \pm 0.02$ \\
\hline $\mathrm{R}-27$ & pass & $2 \min 20 \mathrm{sec}$. & $15.2 \pm 0.03$ & $3.21 \pm 0.02$ \\
\hline $\mathrm{R}-28$ & pass & $1 \mathrm{~min} 37 \mathrm{sec}$. & $16.1 \pm 0.04$ & $3.12 \pm 0.02$ \\
\hline $\mathrm{R}-29$ & pass & $3 \mathrm{~min} 08 \mathrm{sec}$. & $15.1 \pm 0.02$ & $3.16 \pm 0.02$ \\
\hline $\mathrm{R}-30$ & pass & $3 \mathrm{~min} 12 \mathrm{sec}$. & $16.3 \pm 0.04$ & $3.19 \pm 0.01$ \\
\hline
\end{tabular}

All experiments were performed in triplicates (except for the floating lag time) are expressed as mean \pm SD. This result indicates the sustainability of the prepared formulation of cinnarizine hydrochloride.

Raft weight/strength. Table 2 shows the raft weight of different formulations. The formulation (R1) containing the highest percentage of hydroxypropyl cellulose produced the raft of maximum weight (approx. $3.240 \mathrm{~g}$ ). Formulation with the lowest raft weight (approx. $1.974 \mathrm{~g}$ ) was R11 that contained lowest percentage of hydroxypropyl cellulose (Tables 1 and 2). Therefore, it can be concluded that increase in the percentage of release-retardant polymer causes the raft to increase in weight which allows the suspension preparation to be taken at a longer interval. ${ }^{15,16-18}$

Sedimentation volume. The sedimentation volume of the suspensions containing cinnarizine 
hydrochloride was determined using the following formula, $\mathrm{F}$ (sedimentation rate) $=\mathrm{Hu} / \mathrm{H}_{0}$ where the $\mathrm{Hu}$ represents the sediment height and $\mathrm{H}_{0}$ represents the actual height of the suspension (Table 3). Sedimentation rate of all the formulations was found in between $35 \%$ to $100 \%$. Therefore, later we carefully selected eight formulations (R1, R3, R11, R13, R16, R17, R23 and R27) that complied most with the general requirements for suspension preparations (Table 3, Figure 1A). Previous studies suggested that when the sedimentation value, $\mathrm{F}=1$, there will be no sedimentation and no clear supernatant and the suspension is stable; If $\mathrm{F}=0.5$ or $50 \%$, the total volume of suspension is occupied by the sediment; If $F>1$, the sediment volume is greater than the original volume indicating the presence of loose floccules which are mostly fluffy in texture. Therefore, we excluded those having $\mathrm{F}$ values below $80 \% .^{13,14}$

Table 3. Different parameters of the formulated cinnarizine hydrochloride sustained-release suspensions,

\begin{tabular}{cccc}
\hline $\begin{array}{c}\text { Formulation } \\
\text { no. }\end{array}$ & $\begin{array}{c}\text { Sedimentation volume } \\
\mathrm{F}=\mathrm{Hu} / \mathrm{Ho}\end{array}$ & $\begin{array}{c}\text { Degree of flocculation } \\
\beta=\mathrm{F} / \mathrm{F}_{0}\end{array}$ & $\begin{array}{c}\text { Redispersibility } \\
(\%)\end{array}$ \\
\hline R-1 & 0.85 & 0.85 & 95 \\
R-3 & 1.00 & 1.05 & 100 \\
R-11 & 1.00 & 1.00 & 100 \\
R-13 & 0.95 & 1.00 & 95 \\
R-16 & 0.80 & 0.80 & 90 \\
R-17 & 1.00 & 1.01 & 100 \\
R-23 & 0.98 & 1.00 & 100 \\
R-27 & 0.95 & 1.00 & 100 \\
\hline
\end{tabular}

Table 4. The Table represents the data obtained from the one-way ANOVA test results.

\begin{tabular}{lrrrrr}
\hline ANOVA table* & SS & DF & MS & F (DFn, DFd) & P value \\
\hline Treatment (between columns) & 16743 & 7 & 2392 & F $(7,40)=$ & P $<0.0001$ \\
& & & & & 12.21 \\
Residual (within columns) & 7839 & 40 & 196.0 & & \\
Total & 24581 & 47 & & & \\
\hline
\end{tabular}

Data summary**, Number of treatments (columns) $=8$, Number of values (total) $=48$

The test was performed to analyse if significant differences can be reported in the dissolution among all six formulations. *ANOVA was perfumed followed by Tukey's multiple comparisons tests using GraphPad Prism version 8.00. **The dissolution data for formulation R1, R3, R13, R17, R23 and R27.

Degree of flocculation. The degree of flocculation of the suspensions containing cinnarizine hydrochloride was determined using the following formula, $\beta=F / F \infty \beta=(\mathrm{Vu} / \mathrm{Vo}) /(\mathrm{V} \infty / \mathrm{Vo}) \beta=\mathrm{Vu} / \mathrm{V} \infty$ (Table 3, Figure 1B). Theoretically, the minimum value of $B$ is 1 , when the sedimentation volume of the flocculated suspension is equal to the sedimentation volume of deflocculated suspension. Some formulations failed to comply with the requirement of degree of flocculation for pharmaceutical suspensions and we discarded these preparations. Finally, we selected eight formulations (R1, R3, R11, R13, R16,
$\mathrm{R} 17, \mathrm{R} 23$ and R27) that showed the $\mathrm{B}$ value within the acceptable limit of $85-100 \%$ indicating that the suspensions consisted of loose fluffy floccules which are readily redispersible upon manual shaking (approx. 1-2 inversions). ${ }^{14}$

Resuspendability/redispersibility. Each formulation containing cinnarizine suspensions was taken into $100 \mathrm{ml}$ colourless polypropylene bottles and allowed to stand for 4 days at room temperature (approx. $25^{\circ}$ C.) without any shaking. After 4 days on constant standing, each bottle was subjected to manual shaking and the time required for the sediment to 
convert into a homogenous suspension was recorded. One inversion was considered as $100 \%$ easy to be

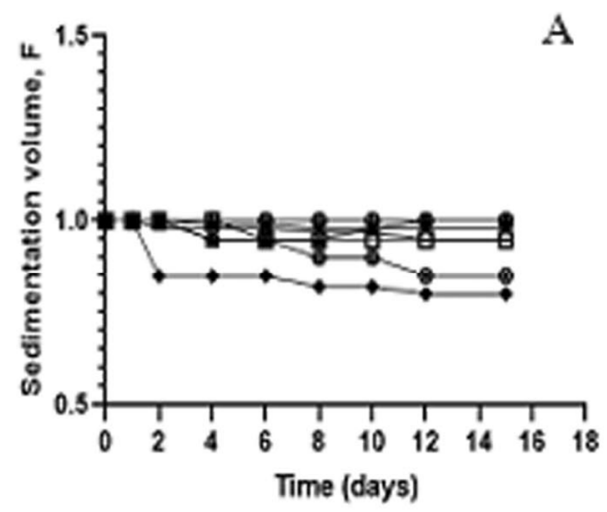

redispersed and every additional inversion decreased the percent ease of redispersibility by $5 \%$ (Table 3 ). ${ }^{15}$

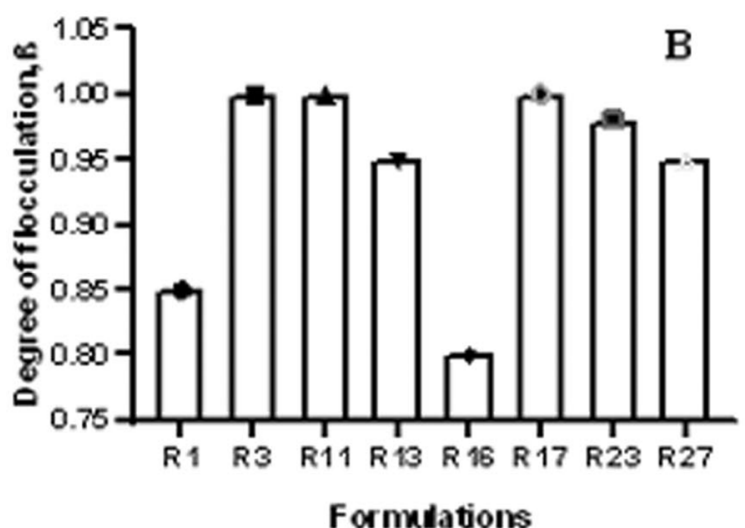

Figure 1 represents the sedimentation volume and the degree of flocculation of the formulated suspensions. A. Sedimentation volume of the selected formulations containing cinnarizine hydrochloride suspension at different time intervals (days). Different shape of the symbols represents the different formulations shown at the top of the graph. B. The degree of flocculation among the selected formulations.

In vitro drug release data. Formulations $\mathrm{R} 1$, R3, R13, R17, R23, and R27 were selected for further characterization based on the results found on the above studies. To perform in vitro dissolution test, we discarded two formulations R11 and R16 due to poor raft weight, low sedimentation volume, lowest degree of flocculation and redispersibility (Table 2 and 3) from the eight previously selected formulations. Thus formulation R1, R3, R13, R17, R23 and R27 were finally selected for the in-vitro drug release studies (Figure 2). The dissolution study was performed with the selected formulations in $0.1 \mathrm{~N}$ $\mathrm{HCl}$ for up to 12 hours and the percentage of drug release was calculated at each time interval $(15 \mathrm{~min}$, $30 \mathrm{~min}, 1,2,4,6,8$ and 12 hours). Cinnarizine content in the collected aliquot was determined spectrophotometrically using double beam UVVisible spectrophotometer (Shimadzu 1700) at a $\lambda$ $\max$ of $251 \mathrm{~nm}$ after suitable dilution of each formulation. ${ }^{15-18}$ Percentage of drug release at each time interval was plotted against time in graphs that demonstrated a marked difference in the dissolution characteristics among different formulations (Figure 3 ). Formulations that showed more than $85 \%$ of drug release in a 12-hour time period, i.e. R1, R3, R13,
R17, R23 and R27 were further studied to assess and compare the drug release kinetics (Figure 3).

The comparison among the 6 formulations containing cinnarizine hydrochloride showed $\geq 85 \%$ drug release in 12 hours. However, the release of cinnarizine from the formulations R1, R3 and R27 were $100.23 \%, 103.83 \%$ and $102.38 \%$ respectively at the end of 12-hours. These data support the desired release profile for controlled/sustained drug delivery up to 12 hours. Besides, these three formulations contained the highest percentage of release-retardant polymer e.g. HPC (Table 1). In contrast, the drug release from other three formulations (R13, R17, and R23) were in the following manner, $98.15 \%, 88.23 \%$ and $90.73 \%$ respectively due to the presence of a low concentration of hydrophilic release-retardant polymers compared to the former formulations. Therefore, the dissolution patterns of the formulations (R1, R3 and R27) demonstrated that the inclusion of a high concentration of hydrophilic release-retardant polymers slowed down the release of drug particles at a time-dependent manner e.g. slow release at initial time followed by a higher rate of release at later hours. The slow-release pattern of the drug at the beginning of the dissolution test was 
Formulation R1

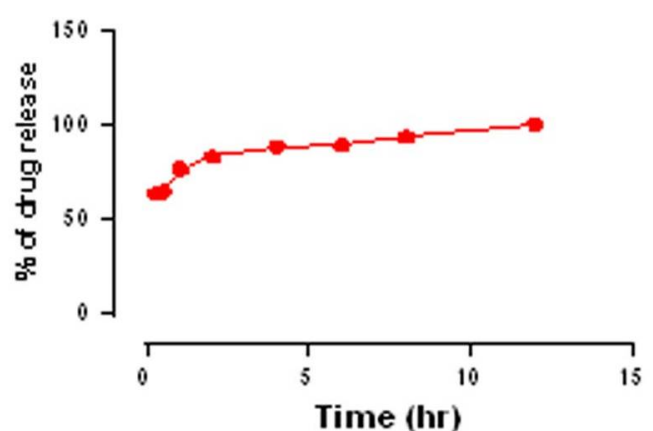

\begin{tabular}{|l|l|}
\hline$r$ & 0.8999 \\
\hline $95 \%$ con fidence interval & 0.5334 to 0.9819 \\
\hline R squared & 0.8097 \\
\hline
\end{tabular}

Formulation R13

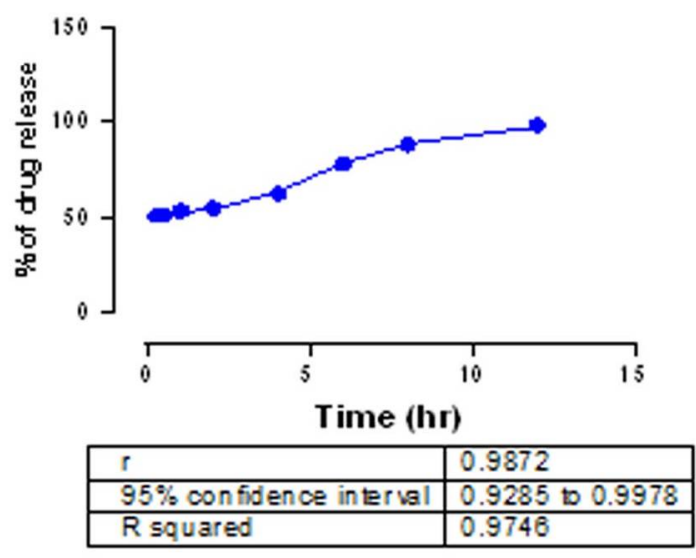

Formulation R23

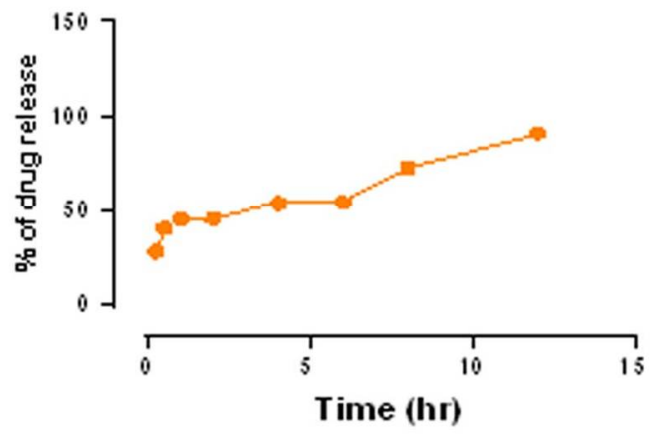

\begin{tabular}{|l|l|}
\hline$r$ & 0.9687 \\
\hline $95 \%$ con fidence interval & 0.8219 to 0.9941 \\
\hline R squared & 0.9345 \\
\hline
\end{tabular}

F ormulation R 3

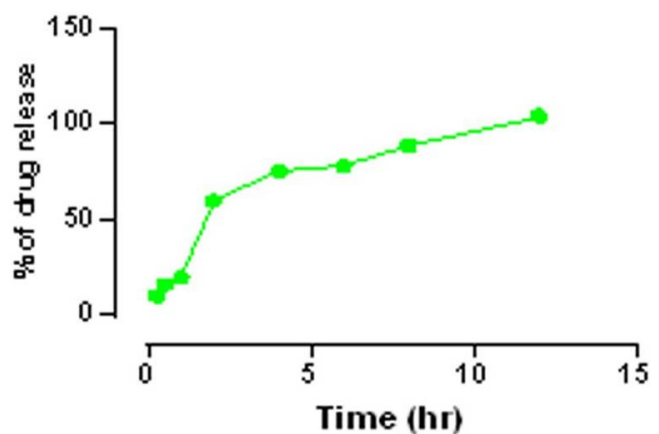

\begin{tabular}{|l|l|}
\hline$r$ & 0.9102 \\
\hline $95 \%$ confidence interval & 0.5731 to 0.9838 \\
\hline$R$ squared & 0.8285 \\
\hline
\end{tabular}

\section{Formulation R 17}

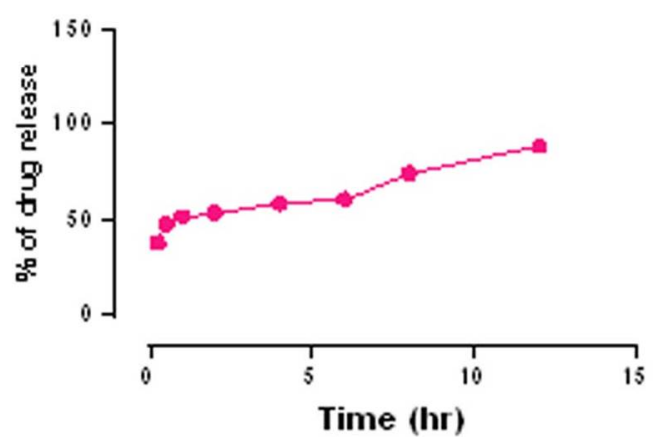

\begin{tabular}{|l|l|}
\hline$r$ & 0.9889 \\
\hline $95 \%$ confiden $\propto$ interval & 0.8327 to 0.9945 \\
\hline$R$ squared & 0.9387 \\
\hline
\end{tabular}

F ormulation R 27

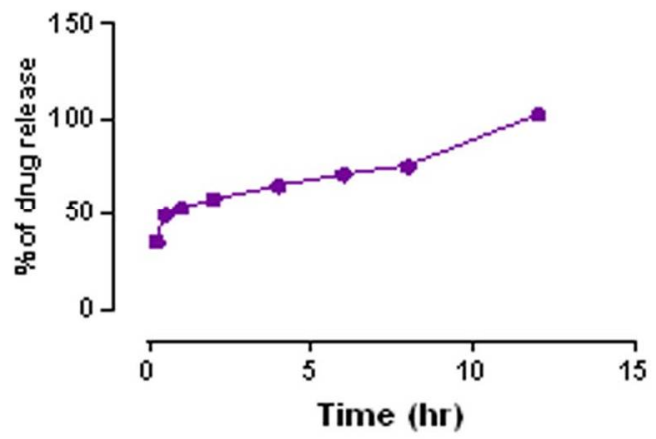

\begin{tabular}{|l|l|}
\hline$r$ & 0.9642 \\
\hline $95 \%$ confiden $\propto$ in terval & 0.8098 to 0.9937 \\
\hline$R$ squared & 0.9298 \\
\hline
\end{tabular}

Figure 2. Dissolution study of cinnarizine hydrochloride sustained-release suspensions (formulation R1, R3, R13, R17, R23, and R27) in $0.1 \mathrm{~N} \mathrm{HCl}$. The data illustrate the percentage of drug release at different time intervals starting from 15 minutes to 12 hours. The curves representing the linearity with an $\mathrm{R}^{2}$ value over $80 \%-97 \%$; correlation coefficient value, $\mathrm{r}$ over $90-98 \%$. 
observed possibly due to the presence of the polymeric substances which retarded the release of the drug. ${ }^{19-23}$ On the other hand, the desired release at 12-hour was observed due to the hydrophilic nature of the release-retardant polymers present in the formulation which started swelling and eventually formed a gel-like mass. ${ }^{22-24}$

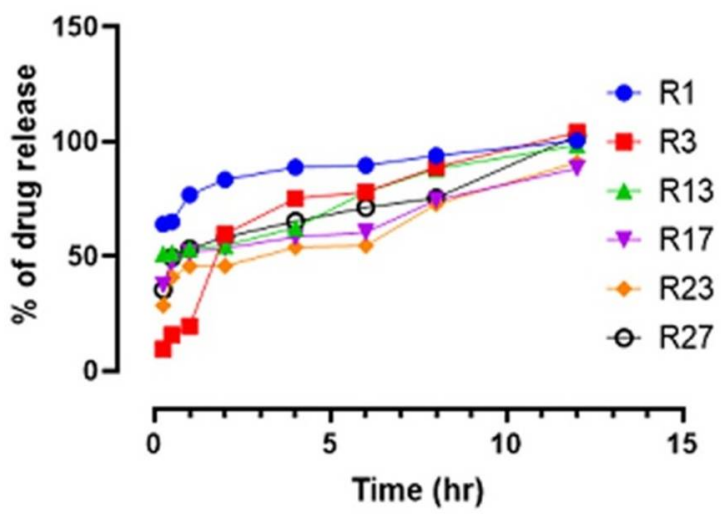

Figure 3. Comparison of cumulative percentage of drug release of hydrochloride sustain release oral suspensions in $0.1 \mathrm{~N} \mathrm{HCl}$ at different time intervals among the selected formulations; formulations are labeled in different shapes of symbols as shown at the right side of the graph.

Statistical analysis among the six formulations in terms of percentage of drug-release at each time point using ANOVA followed by Tukey's multiple comparisons tests showed that formulations R1. R3, R13, R17, R23 and R27 differed significantly, $\mathrm{p}<0.0001$, Table $4 .^{25}$

\section{CONCLUSION}

In-vitro drug release profile is dependent upon the dissolution behaviour and release pattern of the API in the selected dissolution medium. The dissolution profile of a drug can be modified by excipients which may be the release-retardant polymer. The current study demonstrates that drugrelease can be increased in a time-dependent manner by increasing the concentration of release-retardant polymers. We have observed that the raft-forming technique can be efficiently employed to achieving an extended drug-release by using proper concentrations of HPC. Further investigation of the formulated cinnarizine hydrochloride oral suspensions in an in-vivo system would support the effectiveness of the drug delivery method and provide sufficient evidence for advanced IVIVC studies.

\section{ACKNOWLEDGEMENT}

We acknowledge the support of ACI Pharmaceuticals Ltd. for providing the API as a gift.

\section{CONFLICT OF INTEREST}

The authors declare no conflict of interest.

\section{REFERENCES}

1. Foda, N.H., Ali and S.M. 2011. Gastroretentive drug delivery systems as a potential tool for enhancing the efficacy of antibiotics: a review, Int. J. Pharma. Bio Sci. 2, 94-104.

2. Sharma, A. R. and Khan, A, 2014. Gastroretentive Drug Delivery System: An approach to enhance Gastric retention for prolonged drug release. Int. J. Pharm. Sci Res. 5, 10951106.

3. Dixit, N., Maurya, S. D. and Bhanu, P. S. S. 2013. Sustained release drug delivery system; Indian Journal of Research in Pharma. and Biotechnol. 1, 305-310.

4. Bharkatiya, M., Kitawat, S. and Ojha, A. 2014. Floating drug delivery system: a review. J. Drug Deliv. and Therapeut. 4, 130-134

5. Islam, M., Haque, T., Jahangir, R., Fatema, K., Vhuiyan, M. and Biva, I. 2009. In vitro release kinetic study of ciprofloxacin $\mathrm{HCl}$ from Methocel K15M CR, Methocel K4M $\mathrm{CR}$ and Methocel K4M premium matrix tablets. Stamford J. Pharm. Sci. 2, 37-43.

6. Fatema, K., Shahi, S., Shaikh, T. and Zaheer, Z. 2016. Gastroretentive drug delivery system: an overview. Asian Pacific J. Health Sci. 3, 131-144.

7. Yagnesh, T. Naga S. and Kumar R., S. 2016. Pharmaceutical suspensions: patient compliance oral dosage forms. World J. of Pharma. Pharm. Sci. 5, 1471-1537.

8. Arora, S., Ali, J., Ahuja, A., Khar, R. and Baboota, S. 2005. Floating drug delivery systems: A review. AAPS Pharm. Sci. Tech. 6, 372-390.

9. Patel B., Jayvadan P. and Rashmin T. 2010. Improvement of solubility of cinnarizine by using solid dispersion technique. Int. Res. J. Pharm. 1, 127-131.

10. Raghuvanshi, S. and Pathak, K. 2014. Recent advances in delivery systems and therapeutics of cinnarizine: a poorly water-soluble drug with absorption window in stomach. $J$. Drug Deliv. 2014, 1-15.

11. Maghraby, G. M. E., Elzayat, E. M., \&Alanazi, F. K. 2011. Development of modified in-situ gelling oral liquid sustained release formulation of dextromethorphan. Drug Dev. Ind. Pharm. 38, 971-978. 
12. Nagarwal, R., Ridhurkar, D. and Pandit, J. 2010. In vitro release kinetics and bioavailability of gastroretentive cinnarizine hydrochloride tablet. AAPS Pharm. Sci. Tech.11, 294-303.

13. Uddin, M., Mamun, A., Akter, N., Sarwar, M., Rashid, M. and Amran, M. 2016. Pharmacopoeial standards and specifications for pharmaceutical oral liquid preparations. Archi. Curr. Res. Int. 3, 1-12.

14. Brhane, Y., Shibeshi, A. and Gebre M., T. 2014. Evaluation of local gum of Acacia polyacantha as a suspending agent in metronidazole benzoate suspension formulations. Ethiopian Pharm. J. 30, 10-20.

15. Hampson, F., Jolliffe, I., Bakhtyari, A., Taylor, G., Sykes, J., Johnstone, L. and Dettmar, P. 2009. Alginate-antacid combinations: raft formation and gastric retention studies. Drug Dev. Ind. Pharm. 36, 614-623.

16. Jamil, F., Kumar, S., Sharma, S., Vishvakarma, P. and Singh, L. 2011. Review on stomach specific drug delivery systems: development and evaluation. Int. J. Res. Pharm. Biomed. Sci. 2, 1427-1433.

17. Alhamdany, A. T. N., Maraie, N. K. and Msheimsh, B. A. R. 2014. Development and in vitro/in vivo evaluation of floating in situ gelling oral liquid extended release formulation of furosemide. UK J. Pharm. Biosci. 2, 1-11.

18. Prajapati, S., Mehta, A., Modhia, I. and Patel, C. 2012. Formulation and optimisation of raft-forming chewable tablets containing $\mathrm{H}_{2}$ antagonist. Int. J. Pharm. Investigation. 2, 176-182.
19. Golam, M., A., and Syed S., H. 2008. Evaluation of dissolution behavior of paracetamol suspensions, J. Pharm. Sci. 7, 53-58.

20. Torne, S., R., Shila. A. and Sarada., N., C. 2019. Ranitidine controlled release anti-reflux suspension for gastrooesophageal reflux disease and its in-vitro evaluation. Int. J. Appl. Pharm. 11, 74-81.

21. Mandel, D. and Brodie, J. 2000. Review article: alginate-raft formulations in the treatment of heartburn and acid reflux. Aliment. Pharmacol. Therapeut. 14, 669-690.

22. Makarenko, M.V. and Usanov, S. 2014. Floating drug delivery systems: raft forming systems. Proceedings of the Belarusian State University, Series of Physiological, Biochemical and Molecular Biology Sciences. 9, 33-44.

23. Sivaneswari, S., Hemalatha, G., Jayasree, J., Mounika, B., Murthy, S. and Preethi, N. 2014. Role of various natural, synthetic and semi-synthetic polymers on drug release kinetics of losartan potassium oral controlled release tablets. Int. J. Pharm. Inv. 4, 183-188.

24. Poornima, P., Abbulu, K. and Mukkanti, K. 2017. Formulation and in-vitro evaluation of gastro-retentive floating tablets containing quetiapine fumarate. Int. J. Pharm. Sci. and Drug Res. 9, 315-322.

25. Graphpad.com. 2019. GraphPad Statistics Guide. [online] Available at: http://www.graphpad.com/guides/prism/7/ statistics/index.htm. 